'Laboratorio de Sueño, Instituto de Nutrición y

Tecnología de los Alimentos (INTA), Universidad de Chile, Santiago, Chile ${ }^{a}$ Nutricionista; programa de Doctorado Nutrición y Alimentos, Universidad de Chile; Becario CONICYT. ${ }^{b}$ Nutricionista; programa de Magíster en Ciencias de la Nutrición, INTA, Universidad de Chile.

Financiamiento: Proyecto Fondecyt 1110513.

Recibido el 2 de agosto de 2010, aceptado el 11 de abril de 2011.

Correspondencia a: Rodrigo A. Chamorro Melo Laboratorio de Sueño INTA, Universidad de Chile Avda. El Líbano 5524 Macul Santiago, Chile. Tel: (56-2) 9781447

Fax: (56-2) 2214030 E-mail: rchamorro@inta. uchile.cl

\section{La reducción del sueño como factor de riesgo para obesidad}

\author{
RODRIGO A. CHAMORRO ${ }^{1, a}$, SAMUEL A. DURÁN ${ }^{1, a}$, SUSSANNE C. REYES ${ }^{1, a}$, \\ ROSEMARIE PONCE ${ }^{1, \mathrm{~b}}$, CECILIA R. ALGARÍN ${ }^{1}$, PATRICIO D. PEIRANO ${ }^{1}$
}

\section{Sleep deprivation as a risk factor for obesity}

Nocturnal sleep patterns may be a contributing factor for the epidemic of obesity. Epidemiologic and experimental studies have reported that sleep restriction is an independent risk factor for weight gain and obesity. Moreover, sleep restriction is significantly associated with incidence and prevalence of obesity and several nontransmissible chronic diseases. Experimental sleep restriction is related to altered plasma leptin and ghrelin concentrations. Both hormones are directly related to appetite and satiety mechanisms. Also, a higher activity of the orexin/hypocretin system has been reported, as well as changes in glucose metabolism and autonomic nervous system. Some studies indicate that these endocrine changes could be associated with a higher diurnal food intake and preference for energy-dense foods. All these changes could result in a positive energy balance, leading to weight gain and a higher obesity risk in the long term. The present article summarizes the epidemiologic and experimental evidence related to sleep deprivation and higher obesity risk. The possible mechanisms are highlighted.

(Rev Med Chile 2011; 139: 932-940).

Key words: Ghrelin; Leptin; Obesity; Sleep deprivation.
L a obesidad $(\mathrm{OB})$ es un problema relevante de salud pública en todos los grupos etáreos y una epidemia en todo el mundo ${ }^{1}$. En la población infantil, su prevalencia y severidad continúa en aumento, al igual que sus comorbilidades, en particular, las secuelas que involucran los sistemas nervioso central, cardiovascular y metabóli$\mathrm{CO}^{2,3}$. Su prevención resulta un problema urgente, siendo las estrategias alimentario-nutricional y/o de actividad física los principales focos abordados para dicho propósito. Sin embargo, análisis recientes de intervenciones en dichos factores indican éxito principalmente a corto plazo ${ }^{4,5}$.

En este contexto, diversos estudios respaldan la hipótesis que sostiene que la cantidad y calidad del sueño nocturno serían factores relevantes relacionados con la $\mathrm{OB}^{6,7}$. Actualmente, la población de países industrializados ha disminuido su cantidad de sueño nocturno ${ }^{8}$. Datos estadounidenses muestran que los adultos han reducido entre 1 y
2 horas su cantidad de sueño y más de un tercio de los adultos jóvenes refieren dormir $<7$ horas ${ }^{8}$, fenómeno igualmente reportado en niños y adolescentes ${ }^{9,10}$.

Lo anterior es relevante, pues diversos estudios han indicado que la disminución de la cantidad de sueño nocturno sería un factor de riesgo para ganancia de peso y desarrollo de $\mathrm{OB}$ en adultos y niños ${ }^{11,12}$. La presente revisión pretende resumir la evidencia epidemiológica y experimental que respalda el rol del sueño en la OB, con especial énfasis en los mecanismos en los que se sustenta la evidencia actual.

\section{La epidemia de obesidad}

La OB es una patología compleja, resultante de la interacción de factores genéticos y ambientales ${ }^{13}$. Reconocida como una epidemia mundial por la Organización Mundial de la Salud (OMS), afecta 
con particular severidad a la población de países en desarrollo y de menor nivel socioeconómico ${ }^{13}$.

Los datos de prevalencia indican que el problema a nivel mundial es relevante ${ }^{14}$. La estimación de prevalencia de sobrepeso (índice de masa corporal [IMC] 25-29,9) y de OB (IMC $\geq 30)$ en EE.UU. de Norteamérica es de 68 y $32,2 \%$, respectivamente ${ }^{15}$. Aún cuando algunos reportes muestran una estabilización, otros autores indican que el porcentaje de individuos que presentan grados más severos de OB podría mantener una tendencia al $\mathrm{alza}^{16}$. En Chile, la prevalencia de OB al año 2004 en niños pre-escolares era de $20,1 \%$, muestra una tendencia creciente (período 2002-2004) y un incremento en función de la edad ${ }^{17,18}$; en escolares se ha observado una tendencia similar ${ }^{19}$ (Figura 1).

La importancia de la prevención de la $\mathrm{OB}$ es relevante, particularmente a edades tempranas ${ }^{20}$. La OB implica un alto costo directo e indirecto para el individuo y la sociedad, y en población pediátrica se asocia a diversas comorbilidades endocrinas, metabólicas y a un mayor riesgo de $\mathrm{OB}$ en la adultez ${ }^{21}$. Asimismo, es una condición que determina un elevado costo para los sistemas de salud ${ }^{14}$.

\section{Cambios en una sociedad de "24 horas"}

Uno de los cambios comportamentales que se han observado en sociedades industrializadas ha sido la disminución de las horas destinadas al sueño nocturno ${ }^{22}$. La disponibilidad de la luz eléctrica, aparatos tecnológicos, mayor demanda laboral, turnos laborales y viajes transmeridianos, se han asociado con este fenómeno ${ }^{22}$. A modo de ejemplo, adolescentes de distintas culturas utilizan la televisión y el computador diariamente y por períodos prolongados, con el consiguiente retraso en el inicio del período de sueño y reducción del mismo ${ }^{23,24}$. Se ha demostrado que la "cultura de la somnolencia", socialmente aceptada, se asocia con menor rendimiento cognitivo, mayor riesgo de accidentabilidad ${ }^{25}$ y desmedro del estado de salud $^{22}$. Se ha propuesto que la pérdida del orden temporal interno, por ejemplo entre los patrones de sueño-vigilia y alimentación-ayuno, podría resultar en alteraciones metabólicas tempranas conducentes a desórdenes como la $\mathrm{OB}^{26,27}$.

\section{El sueño y sus estados}

Típicamente, el sueño se divide en dos estados: sueño REM (SREM) y sueño no-REM (SNREM), los que ciclan continuamente a través de un período de dormir ${ }^{28}$. Cada estado de sueño representa un modo de funcionamiento cerebral que involucra al conjunto del organismo, y el "gold standard" para su estudio en seres humanos lo constituye el registro polisomnográfico (PSG). Durante el SREM (acrónimo anglosajón de rapid eye movement), además de la actividad electroencefalográfica (EEG) que lo caracteriza, también ocurren movimientos oculares rápidos y atonía muscular. Este estado de sueño ha sido asociado

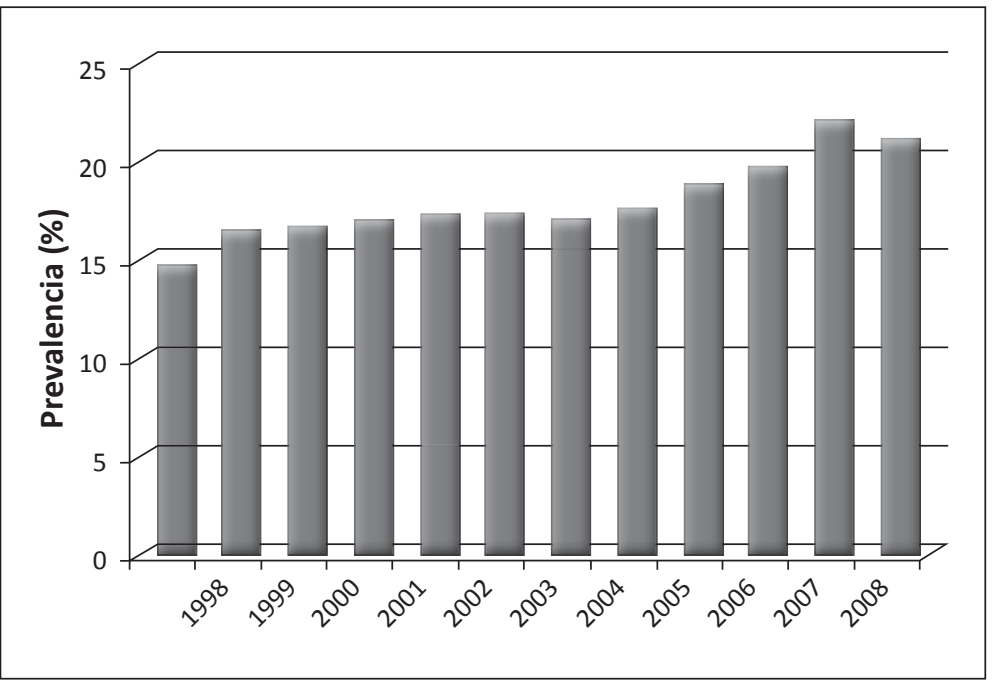

Figura 1. Prevalencia de Obesidad (P/ T>2D.S., según NCHS/OMS) en escolares ( $1^{\circ}$ año básico) chilenos, período 1997-2008. La prevalencia en los años 1997 y 2008 fue 14,4 y 20,8\%, respectivamente [Fuente: Junta Nacional de Auxilio Escolar y Becas (JUNAEB), Julio 2010]. 
con regulación del desarrollo del sistema nervioso, aprendizaje y presencia de ensoñaciones ${ }^{28}$.

En base a la actividad EEG, el SNREM se subdivide en 3 etapas (N1, N2 y N3) ${ }^{29}$ que en orden creciente reflejan la integración del conjunto del encéfalo en frecuencias progresivamente más lentas. La etapa más profunda $\mathrm{N} 3$, se vincula con la restauración somática y la regulación de la liberación hipofisiaria de hormona de crecimiento $^{30}$.

El sueño, tal como la dieta y la actividad física, tiene un rol clave en el crecimiento, desarrollo y mantención del estado de salud, pues participa, entre otros, en la regulación de procesos de aprendizaje, desarrollo cerebral, restauración somática y diversos patrones endocrinos ${ }^{31}$. El principal factor modificador de la cantidad de sueño es la edad ${ }^{32} y$, no obstante la gran variabilidad interindividual, existen referencias respecto de la cantidad diaria para los diferentes rangos etáreos ${ }^{32}$. Así, las horas recomendadas que un(a) menor de 5 años debiera dormir son no menos de 11, para disminuir progresivamente en función de la edad a no menos de 10, 9 y 8 en escolares, adolescentes y adultos, respectivamente ${ }^{33}$.

Entre otros, la actividad metabólica es un proceso modulado a través del sueño. En comparación con la vigilia, el metabolismo energético cerebral (tasa metabólica) disminuye y se modifica en función de sus estados ${ }^{34}$; el consumo de glucosa cerebral es mayor durante la vigilia y el SREM, mientras que alcanza su menor nivel en las etapas profundas del SNREM ${ }^{35}$. Consecuentemente, cambios en la organización temporal (distribución a través del período de sueño) o en la cantidad de sus estados podría resultar en alteraciones metabólicas; en este sentido, un estudio reciente demostró que la etapa N3 de SNREM tendría un rol calve en la regulación del metabolismo de glucosa ${ }^{36}$.

\section{Evidencia epidemiológica que asocia al sueño con la $\mathrm{OB}$}

Además del patrón de ingesta calórica y actividad física, diversos estudios han identificado otros factores involucrados en la epidemia de $\mathrm{OB}^{37}$. Datos observacionales y experimentales recientes brindan respaldo a la hipótesis que sostiene que un inadecuado patrón de sueño (cantidad, calidad, horario) podría contribuir a alteraciones metabólicas tempranas, conducentes a mayor ganancia de peso y riesgo de $\mathrm{OB}$ en diversos grupos etáreos ${ }^{11}$.
La evidencia al respecto se basa en la coincidencia entre el notable aumento en las cifras de $\mathrm{OB} /$ sobrepeso y el aumento en la prevalencia de la llamada "deuda de sueño", caracterizada por el menor tiempo destinado a dormir diariamente y mantenido crónicamente ${ }^{38}$. Estudios epidemiológicos en distintas poblaciones han mostrado que una menor cantidad de sueño se asocia negativamente con el IMC, y determina un mayor riesgo para $\mathrm{OB}^{39}$.

Precisamos que se ha reportado una relación en forma de " $U$ " entre la cantidad de sueño y el IMC en adultos, pues la mayor cantidad de sueño también se asociaría con mayor riesgo para $\mathrm{OB}^{40}$. Estos resultados, sin embargo, podrían reflejar la existencia de trastornos del sueño y no necesariamente el efecto de la cantidad sueño sobre el peso corporal; cabe recordar que la prevalencia de ciertos trastornos del sueño aumenta en función de la edad ${ }^{41}$.

Una asociación inversa entre cantidad de sueño y riesgo para $\mathrm{OB}$ ha sido puesta en evidencia en distintos grupos etáreos ${ }^{42}$. Un estudio de una muestra de 6.800 niños y niñas de 5-6 años, encontró que la prevalencia de $\mathrm{OB}$ y el porcentaje de grasa corporal disminuyeron a mayor duración de sueño, y que el riesgo para ser obeso fue menor (OR 0,45, [IC: 0,28-0,75]) en aquellos niños que cumplieron con sus horas de sueño recomendadas ${ }^{43}$.

Estudios prospectivos en niños han confirmado dichos resultados ${ }^{44,45}$. Touchette et $\mathrm{al}^{46}$, recabaron anualmente información sobre el patrón de sueño y el riesgo para $\mathrm{OB}$ en una muestra de 1.138 niños entre los 2,5 y 6 años de edad, encontrando que quienes durmieron mantenidamente menos de lo recomendado $(<11 \mathrm{~h})$, tuvieron mayor riesgo para ser obesos en la niñez temprana ${ }^{46}$. Más aún, una menor cantidad de sueño a los 10 años de edad aumentó el riesgo de desarrollar $\mathrm{OB}$ en la adultez (a los 30 años), aún después de controlar por diversos factores confundentes ${ }^{47}$.

Un número reducido de estudios han confirmado esa relación utilizado métodos de registro objetivos, mediante la actigrafía (permite registrar la actividad motora e inferir a través de dicha información, los períodos de sueño y de vigilia durante períodos de tiempo prolongados $)^{48} \mathrm{o}$ registro PSG. Una investigación que involucró a 519 niños de 7 años de edad, demostró que menos de 9 horas de sueño fue un factor de riesgo para 
presentar sobrepeso/OB y, además, se asoció a un incremento de 3,3\% en la grasa corporal ${ }^{49}$. En un grupo de 383 adolescentes se reportó una de las asociaciones más potentes entre estas variables: el riesgo para $\mathrm{OB}$ entre 11 y 16 años de edad se incrementó 5 veces por cada hora menos de sueño $^{12}$; investigaciones en poblaciones adulta y adulta-mayor han sido consistentes con dichos hallazgos ${ }^{50,51}$.

La información referida al efecto de la organización de los estados de sueño es menos abundante y consistente. Hace más de tres décadas, algunos estudios ya habían reportado una asociación positiva entre cantidad y porcentaje de SREM y peso corporal en adultos ${ }^{52}$. Más recientemente, Liu et $\mathrm{al}^{53}$ mostraron que el $\mathrm{z}$-score de IMC se asoció negativamente con la cantidad y eficiencia de sueño en niños entre 7 y 17 años -coincidente con hallazgos epidemiológicos previamente descritos-, en particular, con una menor cantidad de SREM y reducida densidad de movimientos oculares rápidos en obesos, pero sin diferencias en relación al SNREM ${ }^{53}$. Esos datos se ven confundidos por la inclusión de sujetos con trastornos emocionales (particularmente, depresión y ansiedad) y un grupo control reducido.

Los cambios en la organización de los estados de sueño, como consecuencia de una restricción y/o detrimento de la calidad del sueño, sugieren que dicha organización podría relacionarse con los mecanismos que median el efecto de la restricción del sueño sobre la ganancia de peso y la OB. Se debe considerar que en su gran mayoría los hallazgos epidemiológicos provienen de la evaluación subjetiva de la cantidad y/o calidad de sueño; en población pediátrica estos provienen de cuestionarios y/o del auto-reporte materno ${ }^{43,46}$. No obstante, diversos meta-análisis han sustentado la evidencia, particularmente en población pediátri$\mathrm{ca}^{54,55} \mathrm{y}$ adultos jóvenes ${ }^{39}$. Finalmente, la mayoría de los estudios han evaluado esta relación con un diseño transversal, impidiendo evaluar el efecto del patrón de sueño sobre la ganancia de peso o el riesgo $\mathrm{OB}$ en el desarrollo, o aproximarse a una relación de causalidad.

\section{Potenciales mecanismos}

A la fecha, se desconocen los mecanismos biológicos precisos que median esta relación ${ }^{56}$. No obstante, diversos estudios realizados en modelos animales y seres humanos han propuesto algunos ${ }^{56}$ (Figura 2). Estos podrían conducir a estimulación de la apetencia, mayor ingesta energética y ganancia de peso temprana.

\section{a) Regulación neuroendocrina y apetito-saciedad}

Se ha propuesto que la relación entre sueño y OB podría relacionarse con un desbalance en el patrón de patrones neuroendocrinos reguladores del apetito y el balance energético ${ }^{57}$.

En una muestra de 11 hombres adultos sanos, expuestos a una restricción ( 4 horas por 6 noches) y extensión del sueño nocturno (12 horas por 6 noches), la concentración plasmática de leptina disminuyó significativamente y la amplitud de su variación dentro de las 24 horas fue $20 \%$ menor cuando los sujetos permanecieron en cama sólo 4 horas/noche ${ }^{70}$. Un estudio posterior confirmó estos hallazgos además de mostrar un incremento de grelina (18\%), utilizando un diseño cross-over, y no obstante reemplazar la ingesta calórica por una infusión constante de glucosa ${ }^{58}$. Además, estas modificaciones endocrinas se asociaron con una mayor sensación de hambre y apetito diurnos (medido en una escala análoga visual), particularmente por alimentos ricos en energía y carbohidratos $^{58}$. Otros autores han mostrado que bastaría una sola noche de privación de sueño para que la concentración plasmática de grelina y la sensación de hambre diurnas aumenten significativamente ${ }^{59}$.

Consistente con esos cambios neuroendocrinos, hombres expuestos a una restricción del sueño presentan una mayor ingesta alimentaria (además de un menor gasto energético relacionado a la actividad física) en condiciones de laboratorio ${ }^{60}$, hallazgos confirmados en un estudio previo ${ }^{61}$, pero no en otro ${ }^{62}$; la divergencia en los resultados probablemente se relaciona a disímiles características de los participantes y un pequeño tamaño muestral. Un estudio epidemiológico de una muestra de 1.024 adultos reportó que una cantidad de sueño reducida $(<8$ horas/noche) se asoció con menor leptinemia $(15,5 \%)$ y mayor grelinemia $(14,9 \%)^{63}$, resultados confirmados posteriormente ${ }^{64}$.

Hallazgos en modelos animales que muestran que la regulación del sueño se afecta durante el desarrollo de $\mathrm{OB}$, sugieren que los mecanismos regulatorios del sueño-vigilia y el peso corporal podrían entrecruzarse ${ }^{65}$. La restricción de sueño podría estar asociada con una modificación de 


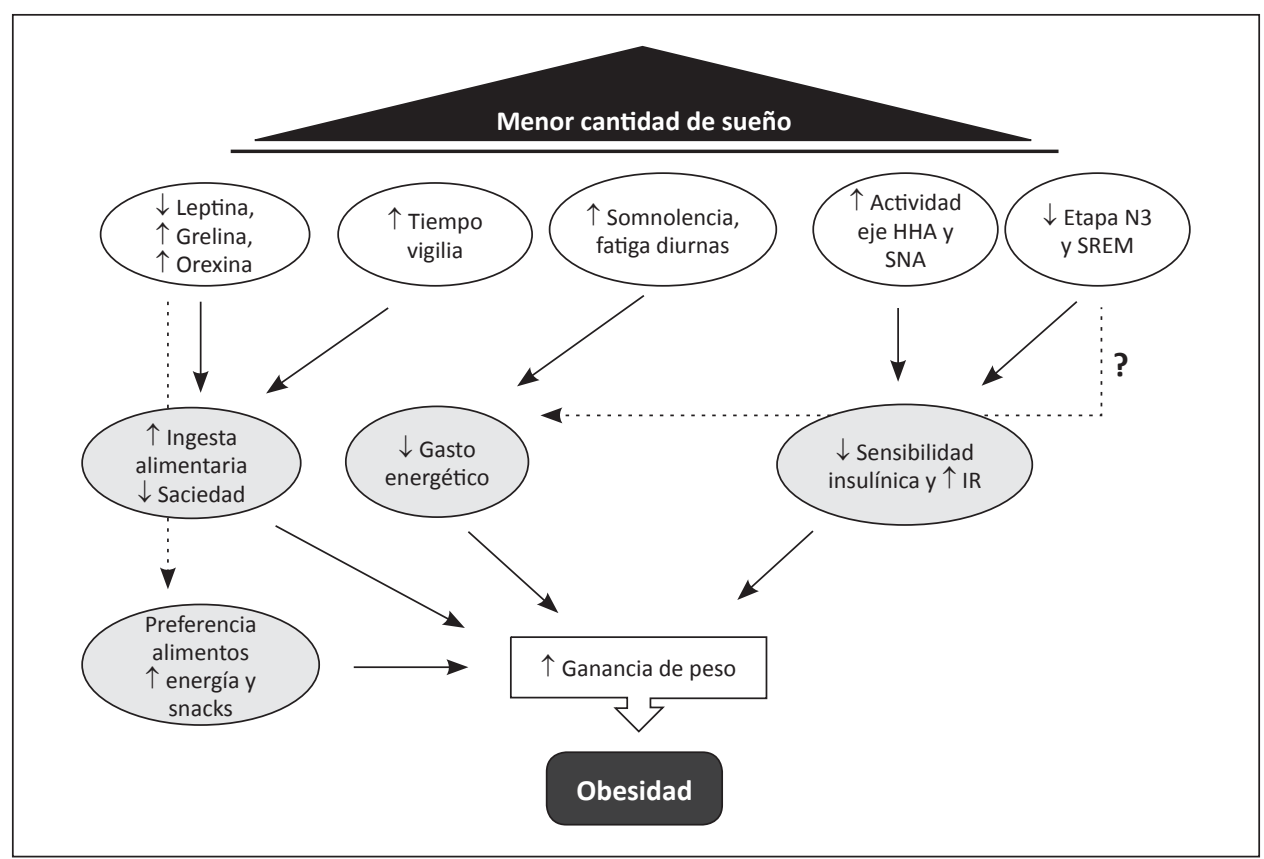

Figura 2. Potenciales mecanismos que relacionan menor cantidad de sueño con ganancia de peso y obesidad. Una menor cantidad de sueño podría resultar en: a) aumento de la concentración plasmática de grelina y de la actividad del sistema orexinérgico hipotalámico, y disminución de leptina, b) menor sensibilidad y mayor resistencia insulínica (RI), c) mayor actividad del sistema nervioso autónomo (SNA) y d) cambios en la organización de los estados de SREM y SNREM; además, una menor cantidad de sueño podría resultar en mayor somnolencia y sensación de fatiga diurnas, mayor actividad del eje HHA, y menor gasto energético. El conjunto de estos cambios podría contribuir a mayor ganancia de peso y riesgo para OB. El rol potencial de los estados de sueño sobre el gasto energético y el metabolismo glucídico es una arista menos explorada a la fecha (línea punteada) [HHA: Hipotálamo-hipófisis-adrenal].

péptidos hipotalámicos reguladores del apetito/ saciedad, en particular al sistema neuropéptido orexina $^{66}$. Este sistema se expresa en el hipotálamo lateral y posterior, una región cerebral clásicamente relacionada con el control del apetito y que evoca la conducta de alimentación en animales ${ }^{67}$, e inerva áreas cerebrales involucradas en la regulación del ciclo sueño-vigilia (CSV) y del sistema nervioso autónomo (SNA) ${ }^{68}$. La privación de sueño aumenta la actividad del sistema orexinérgico, lo que podría promover un mayor tono del sistema nervioso simpático (SNS) y aumentaría la actividad de grupos neuronales estimuladores del apetito (mediados por neuropéptido Y) en el núcleo arcuato hipotalámico ${ }^{69}$.

Los estudios de restricción experimental de sueño sugieren que una alteración de los mecanismos neuroendocrinos relacionados al balance apetito/saciedad, podrían subyacer los efectos del acortamiento del sueño en la ganancia de peso.
Dado que la restricción aguda no es sostenible en el tiempo, es poco evidente de qué forma esos cambios (evidenciado en un reducido número de sujetos) podrían corresponderse con una condición crónica de restricción del sueño, como sería la esperable a nivel poblacional.

\section{b) Metabolismo glucídico}

Spiegel et al., evaluaron a voluntarios jóvenes en una restricción de sueño de $4 \mathrm{~h} /$ noche durante 6 noches consecutivas ${ }^{70}$ y observaron una disminución de la tolerancia a la glucosa (40\%), como consecuencia de una menor utilización y menor respuesta de insulina aguda a la glucosa ${ }^{70}$; estudios posteriores han mostrado resultados concordantes $^{71}$. Investigaciones experimentales recientes han mostrado que la restricción parcial de sueño aumenta la concentración plasmática de insulina e IGF- $1^{72}$, y disminuye la sensibilidad insulínica, luego de tan sólo una noche de restricción de 
sueño en adultos sanos ${ }^{73}$, evidencia apoyada por estudios en roedores ${ }^{74}$.

Además del efecto de la modificación en la cantidad del sueño, cambios en la organización de sus estados podrían modificar la homeostasis de la glucosa. En este sentido, Tasali et al, mostraron que la privación selectiva de la etapa N3 del SNREM durante tres noches y sin alterar la cantidad de sueño, redujo notablemente la sensibilidad insulínica y la tolerancia a la glucosa, induciendo cambios similares a los de un estado pre-diabético ${ }^{36}$. Si bien esa alteración es aguda y en sujetos de sexo masculino, los datos sugieren que la calidad del sueño (en particular la modificación de etapas profundas de SNREM), afecta negativamente los mecanismos que participan en la regulación del metabolismo glucídico. En un contexto de largo plazo, dichos cambios podrían afectar tempranamente su tolerancia y contribuir a mayor riesgo para $\mathrm{DM} 2^{75}$, aún cuando faltan estudios que confirmen esos hallazgos y lo hagan en un período más prolongado de tiempo. Interesantemente, Chaput et al, mostraron que el riesgo ajustado para la incidencia de DM2 y alteración temprana de la tolerancia a la glucosa fue mayor en adultos con una disminuida cantidad de sueño (menos de 7 horas $)^{76}$.

\section{c) Regulación del SNA}

El CSV es el mayor modulador de la actividad autonómica; a modo de ejemplo, el SNREM se asocia con una disminución del SNS y un incremento del tono SNPS respecto de la vigilia ${ }^{77}$, cambios que se revierten parcialmente durante el SREM ${ }^{78}$. En este sentido los cambios en la cantidad o calidad del sueño podrían manifestarse en modificaciones de la regulación del SNA.

Tanto en adultos normotensos ${ }^{79}$ e hiperten$\operatorname{sos}^{80}$, se ha observado que la restricción del sueño aumenta significativamente la presión arterial y la actividad del SNS. Además, la fragmentación del sueño se asocia con un incremento de la actividad adrenocortical y del SNS, cambios que se asocian con una disminución de la sensibilidad a la insulina y de la eficacia de la glucosa ${ }^{81}$. Por otro lado, la restricción experimental del sueño a $4 \mathrm{~h} /$ noche determina una mayor actividad del SNS y concentración de cortisol plasmático (Figura 2) ${ }^{70}$. La hiperactividad simpática y la hipercortisolemia podrían contribuir a afectar negativamente el metabolismo glucídico y mediar los cambios observados en la regulación de leptina e insulina en tejidos periféricos ${ }^{10}$.

Finalmente, la mantención de un balance energético positivo consecuencia de la restricción de sueño, podría ser consecuencia de un mayor nivel de somnolencia, fatiga (contribuyendo a mayor sedentarismo) e ingesta calórica diurnas (a partir de alimentos energéticamente densos). Existe escasa evidencia para sustentar algunos de esos cambios comportamentales ${ }^{60-62}$, requiriéndose estudios experimentales prolongados para confirmar esa hipótesis.

\section{Conclusiones}

Una de las modificaciones comportamentales, cada vez más prevalentes globalmente, es la reducción del tiempo destinado al sueño nocturno. La evidencia actual respalda la hipótesis de que este cambio afecta negativamente la regulación del peso corporal y constituye un factor de riesgo independiente para OB. Consecuentemente, enfatizar la relevancia del sueño podría constituir un factor con impacto potencial a nivel de salud pública en la prevención de la OB. Se hacen necesarios estudios longitudinales en distintos grupos etáreos, corroborar la evidencia experimental y, particularmente, ensayos clínicos controlados que permitan esclarecer una relación causal.

Agradecimientos. Se agradece a Juliana Kain por su ayuda en la elaboración de la Figura 1.

\section{Referencias}

1. World Health Organization. Diet, nutrition and the prevention of chronic diseases. World Health Organ Tech Rep Ser 2003; 916: i-viii, 1-149, backcover.

2. Bessesen DH. Update on obesity. J Clin Endocrinol Metab 2010; 93: 2027-34.

3. Lancet (editorial). The Global Challenge of diabetes. Lancet 2008; 24: 1723.

4. Kamath C, Vickers K, Ehrlich A, McGovern L, Johnson J, Singhal V, et al. Behavioral Interventions to Prevent Childhood Obesity: A Systematic Review and Metaanalyses of Randomized Trials. J Clin Endocrinol Metab 2008; 93: 4606-5.

5. Whitlock E, O’Connor E, Williams S, Beil T, Lutz K. Effectiveness of Weight Management Interventions in Children: A Targeted Systematic Review for the USPSTF. Pediatrics 2010; 125: e396-e418. 
6. Bass J, Turek FW. Sleepless in America: a pathway to obesity and the metabolic syndrome? Arch Intern Med 2005; 165: 15-6.

7. Eisenmann J, Ekkekakis P, Holmes M. Sleep duration and overweight among Australian children and adolescents. Acta Pædiatrica 2006; 95: 956-63.

8. National Sleep Foundation NSF. Sleep in America Poll. 2005.

9. Dollman J, Ridley K, Olds T, Lowe E. Trends in the duration of school-day sleep among 10- to 15-year-old South Australians between 1985 and 2004. Acta Paediatr 2007; 96: 1011-4.

10. Van Cauter E, Knutson KL. Sleep and the epidemic of obesity in children and adults. Eur J Endocrinol 2008; 159 Suppl 1: S59-66.

11. Knutson KL, Van Cauter E. Associations between sleep loss and increased risk of obesity and diabetes. Ann N Y Acad Sci 2008; 1129: 287-304.

12. Gupta NK, Mueller WH, Chan W, Meininger JC. Is obesity associated with poor sleep quality in adolescents? Am J Hum Biol 2002; 14: 762-8.

13. World Health Organization. Obesity: preventing and managing the global epidemic. Report of a WHO consultation. World Health Organ Tech Rep Ser 2000; 894: i-xii, 1-253.

14. Wang Y, Beydoun MA, Liang L, Caballero B, Kumanyika SK. Will all Americans become overweight or obese? Estimating the progression and cost of the US obesity epidemic. Obesity (Silver Spring) 2008; 16: 2323-30 .

15. Flegal KM, Carroll MD, Ogden CL, Curtin LR. Prevalence and trends in obesity among US adults, 1999-2008. JAMA 2010; 303: 235-41.

16. Main ML, Rao SC, O'Keefe JH. Trends in obesity and extreme obesity among US adults. JAMA 2010; 303: 1695.

17. Kain J, Albala C, Garcia F, Andrade M. Obesity in Chilean preschool children: anthropometric evolution and socioeconomic determinants. Rev Med Chile 1998; 126: 271-8.

18. Kain J, Lera L, Rojas J, Uauy R. Obesity among preschool children of Santiago, Chile. Rev Med Chile 2007; 135: 63-70.

19. Kain J, Uauy R, Lera L, Taibo M, Espejo F, Albala C. Evolution of the nutritional status of six years old Chilean children (1987-2003). Rev Med Chile 2005; 133: 101320.

20. Han JC, Lawlor DA, Kimm SY. Childhood obesity. Lancet 2010; 375: 1737-48.

21. Dietz WH. Health consequences of obesity in youth: childhood predictors of adult disease. Pediatrics 1998; 101: 518-25.
22. Rajaratnam SM, Arendt J. Health in a 24 -h society. Lancet 2001; 358: 999-1005.

23. Calamaro CJ, Mason TB, Ratcliffe SJ. Adolescents living the 24/7 lifestyle: effects of caffeine and technology on sleep duration and daytime functioning. Pediatrics 2009; 123: e1005-10.

24. Gibson E, Powles P, Thabane L, O'Brien S, Molnar D, Trajanovic N, et al. "Sleepiness" is serious in adolescence: two surveys of 3235 Canadian students. BMC Public Health 2006; 6: 116.

25. Zee P, Turek F. Sleep and Health. Arch Intern Med 2006; 166: 1686-8

26. Peirano P, Algarín C, Díaz E, Garrido M, Sir T, Albala C. Ciclo sueño-vigilia y obesidad. Rev Chil Nutr 2000; 27: 162-8.

27. Kalsbeek A, Kreier F, Fliers E, Sauerwein HP, Romijn JA, Buijs RM. Minireview: Circadian control of metabolism by the suprachiasmatic nuclei. Endocrinology 2007; 148: 5635-9.

28. McCarley RW. Neurobiology of REM and NREM sleep. Sleep Med 2007; 8: 302-30.

29. Silber M, Ancoli-Israel S, Bonnet M, Chokroverty S, Grigg-Damberger M, Hirshkowitz M, et al. The visual scoring of sleep in adults. J Clin Sleep Med 2007; 3: 12131.

30. Van Cauter E, Spiegel K. Regulation of sleep and circadian rhythms. In: Turek FW, Zee P, eds. Circadian and sleep control of hormonal secretion. New York: Basel, 1999; 397-425.

31. Peirano P, Algarín, C. Sleep in brain development. Biol Res 2007; 40: 471-8.

32. Ohayon MM, Carskadon MA, Guilleminault C, Vitiello MV. Meta-analysis of quantitative sleep parameters from childhood to old age in healthy individuals: developing normative sleep values across the human lifespan. Sleep 2004; 27: 1255-73.

33. Iglowstein I, Jenni OG, Molinari L, Largo RH. Sleep duration from infancy to adolescence: reference values and generational trends. Pediatrics 2003; 111:302-7.

34. Boyle PJ, Scott JC, Krentz AJ, Nagy RJ, Comstock E, Hoffman C. Diminished brain glucose metabolism is a significant determinant for falling rates of systemic glucose utilization during sleep in normal humans. J Clin Invest 1994; 93: 529-35.

35. Nofzinger E, Buysse D, Miewald J, Meltzer C, Price J, Sembrat R, et al. Human regional cerebral glucose metabolism during non-rapid eye movement sleep in relation to waking. Brain 2002; 125: 1105-15.

36. Tasali E, Leproult R, Ehrmann DA, Van Cauter E. Slowwave sleep and the risk of type 2 diabetes in humans. Proc Natl Acad Sci 2008; 105: 1044-9. 
37. Keith SW, Redden DT, Katzmarzyk PT, Boggiano MM, Hanlon EC, Benca RM, et al. Putative contributors to the secular increase in obesity: exploring the roads less traveled. Int J Obes (Lond) 2006; 30: 1585-94.

38. McAllister EJ, Dhurandhar NV, Keith SW, Aronne LJ, Barger J, Baskin M, et al. Ten putative contributors to the obesity epidemic. Crit Rev Food Sci Nutr 2009; 49: 868-913.

39. Nielsen LS, Danielsen KV, Sorensen TI. Short sleep duration as a possible cause of obesity: critical analysis of the epidemiological evidence. Obes Rev 2011; 12: 78-92.

40. Buxton OM, Marcelli E. Short and long sleep are positively associated with obesity, diabetes, hypertension, and cardiovascular disease among adults in the United States. Soc Sci Med 2010; 71: 1027-36.

41. Knutson KL, Turek FW. The U-shaped association between sleep and health: the 2 peaks do not mean the same thing. Sleep 2006; 29: 878-9.

42. Patel SR, Hu FB. Short sleep duration and weight gain: a systematic review. Obesity (Silver Spring) 2008; 16: 643-53.

43. von Kries R, Toschke AM, Wurmser H, Sauerwald T, Koletzko B. Reduced risk for overweight and obesity in 5- and 6-y-old children by duration of sleep-a crosssectional study. Int J Obes Relat Metab Disord 2002; 26: 710-6.

44. Marshall NS, Glozier N, Grunstein RR. Is sleep duration related to obesity? A critical review of the epidemiological evidence. Sleep Med Rev 2008; 12: 289-98.

45. Taveras EM, Rifas-Shiman SL, Oken E, Gunderson EP, Gillman MW. Short sleep duration in infancy and risk of childhood overweight. Arch Pediatr Adolesc Med 2008; 162: 305-11.

46. Touchette E, Petit D, Tremblay RE, Boivin M, Falissard B, Genolini C, et al. Associations between sleep duration patterns and overweight/obesity at age 6. Sleep 2008; 31: 1507-14.

47. Landhuis CE, Poulton R, Welch D, Hancox RJ. Childhood sleep time and long-term risk for obesity: a 32-year prospective birth cohort study. Pediatrics 2008; 122: 955-60.

48. Acebo C, Sadeh A, Seifer R, Tzischinsky O, Wolfson AR, Hafer A, et al. Estimating sleep patterns with activity monitoring in children and adolescents: how many nights are necessary for reliable measures? Sleep 1999; 22: 95-103.

49. Nixon GM, Thompson JM, Han DY, Becroft DM, Clark PM, Robinson E, et al. Short sleep duration in middle childhood: risk factors and consequences. Sleep 2008; 31: 71-8.

50. Lauderdale DS, Knutson KL, Rathouz PJ, Yan LL, Hulley
SB, Liu K. Cross-sectional and longitudinal associations between objectively measured sleep duration and body mass index: the CARDIA Sleep Study. Am J Epidemiol 2009; 170: 805-13.

51. van den Berg JF, Knvistingh Neven A, Tulen JH, Hofman A, Witteman JC, Miedema H. Actigraphic sleep duration and fragmentation are related to obesity in the elderly: the Rotterdam Study. Int J Obes (Lond) 2008; 32: 108390.

52. Adam K. Total and percentage REM sleep correlate with body weight in 36 middle-aged people. Sleep 1987; 10: 69-77.

53. Liu X, Forbes EE, Ryan ND, Rofey D, Hannon TS, Dahl RE. Rapid eye movement sleep in relation to overweight in children and adolescents. Arch Gen Psychiatry 2008; 65: 924-32.

54. Cappuccio FP, Taggart FM, Kandala NB, Currie A, Peile E, Stranges S. Meta-analysis of short sleep duration and obesity in children and adults. Sleep 2008; 31: 619-26.

55. Chen X, Beydoun MA, Wang Y. Is sleep duration associated with childhood obesity? A systematic review and meta-analysis. Obesity (Silver Spring) 2008; 16: 265-74.

56. Knutson KL, Spiegel K, Penev P, Van Cauter E. The metabolic consequences of sleep deprivation. Sleep Med Rev 2007; 11: 163-78.

57. Knutson KL. Impact of sleep and sleep loss on glucose homeostasis and appetite regulation. Sleep Med Clin 2007; 2: 187-97.

58. Spiegel K, Tasali E, Penev P, Van Cauter E. Brief communication: Sleep curtailment in healthy young men is associated with decreased leptin levels, elevated ghrelin levels, and increased hunger and appetite. Ann Intern Med 2004; 141: 846-50.

59. Schmid SM, Hallschmid M, Jauch-Chara K, Born J, Schultes B. A single night of sleep deprivation increases ghrelin levels and feelings of hunger in normal-weight healthy men. J Sleep Res 2008; 17: 331-4.

60. Brondel L, Romer MA, Nougues PM, Touyarou P, Davenne D. Acute partial sleep deprivation increases food intake in healthy men. Am J Clin Nutr 2010; 91: 1550-9.

61. Nedeltcheva AV, Kilkus JM, Imperial J, Kasza K, Schoeller DA, Penev PD. Sleep curtailment is accompanied by increased intake of calories from snacks. Am J Clin Nutr 2009; 89: 126-33.

62. Schmid SM, Hallschmid M, Jauch-Chara K, Wilms B, Benedict C, Lehnert H, et al. Short-term sleep loss decreases physical activity under free-living conditions but does not increase food intake under time-deprived laboratory conditions in healthy men. Am J Clin Nutr 2009; 90: 1476-82.

63. Taheri S, Lin L, Austin D, Young T, Mignot E. Short sleep 
duration is associated with reduced leptin, elevated ghrelin, and increased body mass index. PLoS Med 2004; 1: e62.

64. Chaput JP, Despres JP, Bouchard C, Tremblay A. Short sleep duration is associated with reduced leptin levels and increased adiposity: Results from the Quebec family study. Obesity (Silver Spring) 2007; 15: 253-61.

65. Guan Z, Vgontzas AN, Bixler EO, Fang J. Sleep is increased by weight gain and decreased by weight loss in mice. Sleep 2008; 31: 627-33.

66. Prinz P. Sleep, appetite, and obesity--what is the link? PLoS Med 2004; 1: e61.

67. Adamantidis A, de Lecea L. The hypocretins as sensors for metabolism and arousal. J Physiol 2009; 587: 33-40.

68. Willie JT, Chemelli RM, Sinton CM, Yanagisawa M. To eat or to sleep? Orexin in the regulation of feeding and wakefulness. Annu Rev Neurosci 2001; 24: 429-58.

69. Estabrooke IV, McCarthy MT, Ko E, Chou TC, Chemelli $\mathrm{RM}$, Yanagisawa $\mathrm{M}$, et al. Fos expression in orexin neurons varies with behavioral state. J Neurosci 2001; 21: 1656-62.

70. Spiegel K, Leproult R, Van Cauter E. Impact of sleep debt on metabolic and endocrine function. Lancet 1999; 354: 1435-9.

71. Spiegel K, Knutson K, Leproult R, Tasali E, Van Cauter E. Sleep loss: a novel risk factor for insulin resistance and Type 2 diabetes. J Appl Physiol 2005; 99: 2008-19.

72. van Leeuwen WM, Hublin C, Sallinen M, Harma M, Hirvonen A, Porkka-Heiskanen T. Prolonged sleep restriction affects glucose metabolism in healthy young men. Int J Endocrinol (en prensa 2011).

73. Donga E, van Dijk M, van Dijk JG, Biermasz NR, Lammers GJ, van Kralingen KW, et al. A single night of partial sleep deprivation induces insulin resistance in multiple metabolic pathways in healthy subjects. J Clin Endocrinol Metab 2010; 95: 2963-8.

74. Barf RP, Meerlo P, Scheurink AJ. Chronic sleep disturbance impairs glucose homeostasis in rats. Int J Endocrinol (en prensa 2011).

75. Spiegel K, Tasali E, Leproult R, Van Cauter E. Effects of poor and short sleep on glucose metabolism and obesity risk. Nat Rev Endocrinol 2009; 5: 253-61.

76. Chaput JP, Despres JP, Bouchard C, Astrup A, Tremblay A. Sleep duration as a risk factor for the development of type 2 diabetes or impaired glucose tolerance: analyses of the Quebec Family Study. Sleep Med 2009; 10: 91924.

77. Ferri R, Franceschini C, Zucconi M, Vandi S, Poli F, Bruni $\mathrm{O}$, et al. Searching for a marker of REM sleep behavior disorder: submentalis muscle EMG amplitude analysis during sleep in patients with narcolepsy/cataplexy. Sleep 2008; 31: 1409-17.

78. Kuo TB, Shaw FZ, Lai CJ, Yang CC. Asymmetry in sympathetic and vagal activities during sleep-wake transitions. Sleep 2008; 31: 311-20.

79. Lusardi P, Mugellini A, Preti P, Zoppi A, Derosa G, Fogari R. Effects of a restricted sleep regimen on ambulatory blood pressure monitoring in normotensive subjects. Am J Hypertens 1996; 9: 503-5.

80. Lusardi P, Zoppi A, Preti P, Pesce RM, Piazza E, Fogari R. Effects of insufficient sleep on blood pressure in hypertensive patients: a 24-h study. Am J Hypertens 1999; 12: 63-8.

81. Stamatakis KA, Punjabi NM. Effects of sleep fragmentation on glucose metabolism in normal subjects. Chest 2009; 137: 95-101. 